AIAA-2002-3482

\title{
PRECISION SAR TARGETING USING STEREOSCOPY AND RGPS ABOARD AN UAV
}

\author{
K.A. Beals, R.T. Snider, J.A. Broesch, M.G. Barre, S.J. Scher, and M.W. Funk \\ General Atomics, Photonics, 10240 Flanders Court, San Diego, California 92121-2901
}

\begin{abstract}
An emerging need for timely and precise poor weather targeting in support of long range weapons is identified. A conceptual system capable of all-weather imaging, target identification, target location, and precision guidance is described. This system is largely an integration of currently available technologies that can dramatically increase the accuracy of guided munitions that utilize the Global Positioning System (GPS). The central element of the system is a high-performance SAR that can quickly localize a target and take two 4-in. resolution spotlight images, within a large performance envelope. The two images are taken from the same aspect, with the single exception of a measured squint angle variance. This variance produces a distinct layover difference that can be exploited with stereoscopic (stereo) SAR algorithms to solve the range/height ambiguity and generate three-dimensional coordinate maps of a composite, orthorectified image of the target scene. These coordinates are subsequently relayed to a standard GPS-guided munition, modified to incorporate a datalink. A specially designed Relative GPS (RGPS) algorithm accounts for the separate navigation solution spaces of the sensor and the weapon and solves for GPS positions that maximize the commonality of the bias errors between the two platforms. Flight testing of the stereo SAR targeting and mock relative guidance show promising results.
\end{abstract}

\section{INTRODUCTION}

Recent conflicts, particularly the action in Kososvo, have highlighted the urgent need for timely targeting information and precision guidance of munitions in poor weather. Targeting information from national assets requires significant amounts of time to collect and disseminate to strike forces. In many cases, more than $24 \mathrm{~h}$ can pass between the time a target is recognized and when a guided munition can be released. The situation worsens in poor weather. While GPS guided weapons, such as the joint direct attack munition (JDAM), have been used successfully during poor weather against stationary targets, significant limitations remain. The current $16 \mathrm{~m}$ (SEP) specified accuracy or 8 to $10 \mathrm{~m}$ (CEP) observed accuracy of the GPS Precise Positioning Service (PPS) ${ }^{1}$ coupled with the large damage radius of the JDAM incur a significant risk of undesirable collateral damage.

The idea of coupling synthetic aperture radar (SAR) to a munition using relative GPS (RGPS) to accomplish precision strikes is not new. ${ }^{2}$ The concept of using stereoscopic mensuration in radar grammetry to derive three dimensional coordinates from radar images has been around even longer. ${ }^{3}$ Advances in radar technology have only recently made it possible to sufficiently reduce the size of high-performance SAR sensors to couple these ideas and extend them to a UAV platform. The absolute performance of stereo SAR geolocation is simultaneously improved by reducing the range to the target and increasing resolution at the target. Targeting accuracy can be further enhanced by properly connecting the sensor navigation solution to that of the munition with the use of RGPS. A UAV that is controlled by tactical forces, equipped with a high-resolution SAR, and a munition-capable data link, can significantly reduce the time to strike while simultaneously increasing the performance of GPS guided munitions to precision accuracy (less than $3 \mathrm{~m}$ CEP). This system would expand the current capability to provide precision strikes against stationary, to include relocatable and mobile targets in all weather with inexpensive weapons and greatly reduce the risk of collateral damage for similar mission results. 


\section{APPROACH}

Currently there is no long range operational system that can make precision attacks against most time critical targets (those ground targets that must be engaged shortly after detection) without designation from nearby ground units. What follows is the description of a conceptual system that would enable precision engagement of relocatable and mobile targets that remain stationary for at least half an hour. This system is primarily an integration of existing technology that could be developed rapidly into an operational capability. Flight testing and preliminary results for many of the critical components will be described in the next section.

Mission parameters, intelligence, or cues from theater or ground-based assets provide an area to concentrate a search by a UAV with a high-performance SAR. Mission planning and UAV control software operated by tactical forces on the ground or at sea adjust the UAV flight path to incorporate incoming cues. A lowerresolution strip map $(1 \mathrm{~m}$ or $1 \mathrm{ft})$ is monitored for potential areas of interest by a sensor operator. By selecting a portion of the image in real-time, the operator causes the SAR to automatically switch to spotlight mode and produce a 4-in. resolution image, often without adjusting the UAV flight path. If the operator finds the image sufficiently interesting he will forward it for engagement authorization. The UAV simultaneously adjusts its flight path to set up a second spotlight image with the same aperture center, but an offset ground track. With the second image in hand, the sensor operator can process the two images with a stereoscopic algorithm to generate a three dimensional coordinate for the target. This result is sent on for weapon release planning and sent back to the UAV to prepare for relative guidance of the incoming weapon. Depending upon the ROE and the delay for firing authorization and weapon release the UAV may use a separate sensor to identify the target or perform another " $\mathrm{X}$ " maneuver to refine/update the target coordinate. Once the weapon comes into communication range the weapon and UAV exchange GPS information, the latest target coordinates are transformed into an optimized relative solution space and transmitted to the weapon. The weapon uses this information to guide towards a location which contains bias errors that are similar to those of the sensor.

\section{Stereo SAR}

Stereo SAR algorithms typically measure either crossrange or range layover differences. Layover comes from the ambiguity that results from projecting a three dimensional surface upon a two-dimensional image plane. Suppose the SAR generates spherical wave traveling along a 45-deg depression angle $(\psi)$ that simultaneously intersects both the top edge of a building and the ground in front of the building. If the squint angle $(\theta)$ is $90 \mathrm{deg}$, then the reflected energy from the building top will be recorded on the SAR image at the point on the ground as if it were laid over on the ground. Figure 1 shows how layover works with squinted images. The layover occurs in the direction perpendicular to the ground track of the sensor. The layover is often broken into orthogonal components along the range $\left(l_{\mathrm{r}}\right)$ and crossrange $\left(1_{\mathrm{x}}\right)$ directions, with the range direction defined along the boresight of the SAR at aperture center.

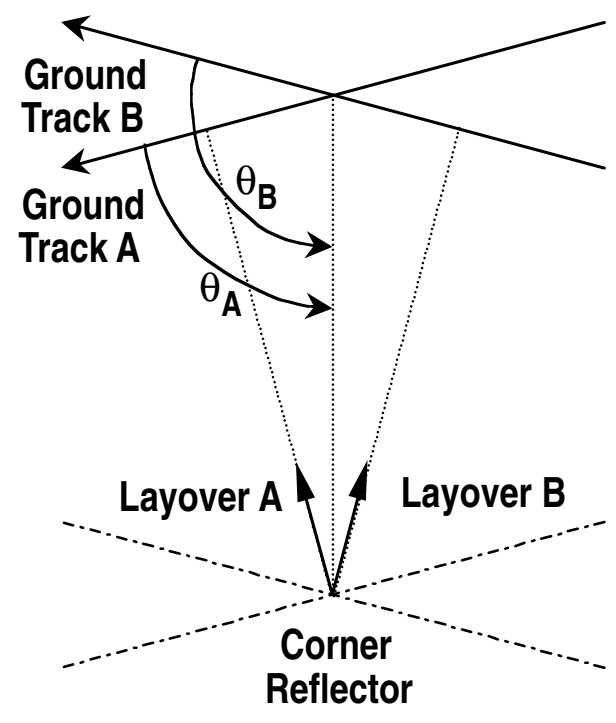

Fig. 1. Cross ground track layover.

One strategy for rapidly searching an area of interest is to make overlapping strip maps. If sequential strips overlap half the width of the previous strip, a continuous stereo survey can be conducted. Image pairs taken with this strategy are collected with a distinct 
depression angle difference that causes range layover differences. Image pairs collected with the geometry depicted in Fig. 1 - ground tracks that cross at aperture center - will exhibit cross range layover differences. Equations (1) and (2) show that either scheme can provide layover differences that are directly proportional to the height (h) of objects within the scene.

$$
\begin{aligned}
& \Delta l_{r}=h\left[\tan \left(\psi_{1}\right)-\tan \left(\psi_{2}\right)\right], \\
& \Delta l_{x}=h\left[\tan \left(\psi_{1}\right) \tan \left(\frac{\pi}{2}-\theta_{1}\right)-\tan \left(\psi_{2}\right) \tan \left(\frac{\pi}{2}-\theta_{2}\right)\right] .
\end{aligned}
$$

Although the crossed track geometry is at a disadvantage for collecting large quantities of data, it has the distinct advantage of keeping the same boresight angle at center aperture for both images. Neglecting misalignment due to navigational error, the two images will be identical except for the crossrange layover. This is critical for precision targeting applications, where accuracy is paramount. In SAR imagery the reflectivity of many scenes depends heavily upon the imaging aspect angle. Figure 2 demonstrates the change in cross range layover and the similarity in boresight angle between two stereo pairs. The pair on the left was imaged with a ground track separation angle of $40 \mathrm{deg}$, while the right pair was taken with an 80 deg angle. Note the similarity of the shadow and ground terrain in each image because of the nearly identical boresights. The reflected energy of the tree's leaves is placed in the foreground, and has an elevation-dependent shift from right to left depending upon the relative ground track for each image. Besides providing a cleaner comparison of reflected magnitude, the nearly identical boresight also opens the potential for interfering the two images phase information. Interferometric SAR (IFSAR) has two benefits. It provides an independent means of calculating object height, and it can be used to aid automatic registration algorithms designed to bring two images into correspondence. $^{4}$

Merely planning to take both images from identical aspect angles is insufficient to guarantee interferometric success. Interferometry depends upon maintaining coherence between the two images across the misalignment distance (d). Equation (3), derived from a

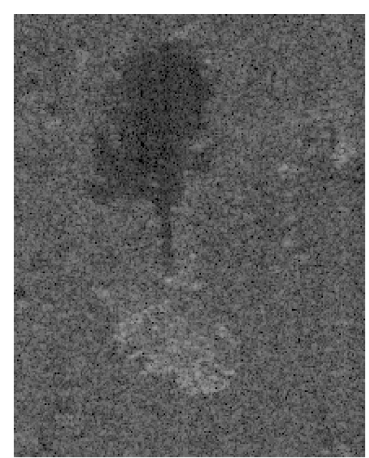

40 degree

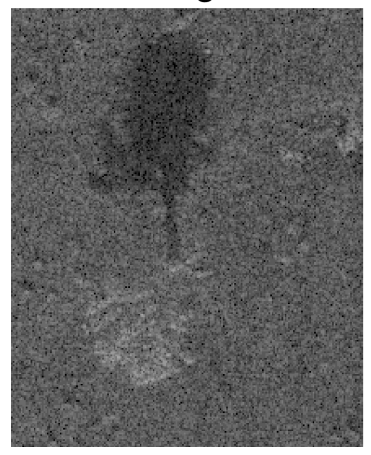

Fig. 2. Stereo pair comparison of a tree.

similar equation for crossed orbital tracks, ${ }^{5}$ provides an approximate upper bound for expected coherence given the slant range $(r)$, radar wavelength $(\lambda)$, and antenna length (a):

$d<\frac{r \lambda}{a}$.

For Ku-band SAR with a small antenna from $10 \mathrm{~km}$ this works out to acceptable misalignments of up to $600 \mathrm{~m}$ or an angular displacement of about $3.5 \mathrm{deg}$. In related work concerning Coherent Change Detection (CCD) performance, GA and SNL have shown strong coherence below $0.5 \mathrm{deg}$ which is the SNL guideline for good CCD performance. GA performed an experiment to examine CCD performance out to $5 \mathrm{deg}$ separation. The general conclusion is that good results can be generated out to $2 \mathrm{deg}$ of displacement. For practical considerations the performance should improve with boresight angle alignment.

Processing a stereo image pair typically produces two products, an orthorectified image of the correlated area and a digital elevation map (DEM) of the same area. An orthorectified image is a composite image that corrects 
for the layover effects found during stereo correlation of the stereo pair. The DEM is pixel registered to the orthorectified image with each pixel value representing the average height determined through stereo and/or IFSAR calculations. One source of error common to both products comes from correlation accuracy when determining the crossrange layover difference. ${ }^{4}$ Minimizing this error leads to a trade off between resolution and accuracy that causes a reduction in resolution of the stereo products with respect to the original image pair. A typical case would be to generate $1-\mathrm{ft}$ resolution products from a 4-in. resolution pair.

\section{Relative Guidance}

There are several levels of GPS positioning accuracy. Standard Positioning Service (SPS) is the most widely available and the least accurate; it degrades significantly from PPS in cases where Selective Availability (SA) is active. As previously noted PPS, or a $\mathrm{P}(\mathrm{Y})$-code based solution, has an observed accuracy of 7-10 m CEP. PPS requires a military grade receiver that is capable of performing $\mathrm{P}(\mathrm{Y})$ decryption.

Differential GPS (DGPS) is the technique of placing a base receiver at a surveyed location and using it to solve for corrections of various measurements made from Space Vehicles (SV) currently in view. These corrections are then sent to a separate receiver, the rover, either in real time or during post-processing. Depending upon the specifics of how DGPS is implemented it can provide results from $5 \mathrm{~m}$ CEP down to millimeter level accuracy. DGPS can be subdivided into three groups: standard code DGPS, carrier-smoothed code DGPS, and carrier phase DGPS. Standard code-based DGPS is simply the basic DGPS. Positions are based upon a least-squares solution derived from pseudorange $(\rho)$ measurements augmented with corrections from the base receiver. The pseudo-ranges are code-based time delays, measured from phase differences in either the Coarse Acquisition (C/A) or Precise (P) codes. Codebased DGPS accuracy typically falls between 1 and $5 \mathrm{~m}$ CEP, depending upon a number of factors including the distance between the base and the rover, the quality of the two receivers, and SV geometry. Unlike standard code-based DGPS, the other DGPS methods depend upon carrier phase $(\phi)$ measurements to achieve higher accuracy. Higher end receivers track the carrier phase of SV signals. These measurements typically have noise that is one to two orders of magnitude less than the pseudo-range code measurements. Carrier phase DGPS uses many epochs of these measurements to converge on an extremely precise solution that is typically accurate from 30 centimeters CEP down to the millimeter level. Carrier-smoothed code DGPS is similar to standard code DGPS with the exception that the cleaner carrier phase measurements are used to smooth the pseudorange measurements used within the leastsquares solution. Carrier-smoothed code solution accuracies range from 3-0.5 m CEP. Equation (4) demonstrates a simple recursive method of smoothing pseudoranges with carrier phase.

$\bar{\rho}\left(t_{i}\right)=\frac{\rho\left(t_{i}\right)+(M-1)\left\{\bar{\rho}\left(t_{i-1}\right)+\lambda\left[\phi\left(t_{i}\right)-\phi\left(t_{i-1}\right)\right]\right\}}{M}$,

where $t_{i}$ represents the current epoch, $t_{i-1}$ is the previous epoch, $M$ is an adjustable filter length, and $\lambda$ is the carrier wavelength. ${ }^{6}$

RGPS can be very similar to DGPS in many ways. The exception is that RGPS does not rely on a base receiver at a known location. Instead RGPS relies on the commonality of bias-like errors to cancel between two or more receivers of interest. Each sub-division of DGPS has a relative analog. For code-based and carriersmoothed code RGPS, simply using the same algorithm on the same SV set at the same time should give very similar results to the DGPS analog. It takes special circumstances, however, to solve for position solutions directly via carrier phase measurements (i.e. to use carrier phase DGPS or RGPS). These circumstances include continuous communications between receivers, continuous phase lock on the same five or more SVs at each receiver, and sufficient time to converge to a solution. In navigation situations where these circumstances are unattainable or not sufficiently reliable, carriersmoothed code solutions may be preferable. Figure 3 shows the results for a zero-baseline, carrier-smoothed code RGPS case with two identical receivers. The plot shows the $\mathrm{X}$ component of ECEF position subtracted from the surveyed location of a rooftop antenna. Both receivers collected data simultaneously from the same antenna for $1.5 \mathrm{~h}$. Note that the two receivers' 


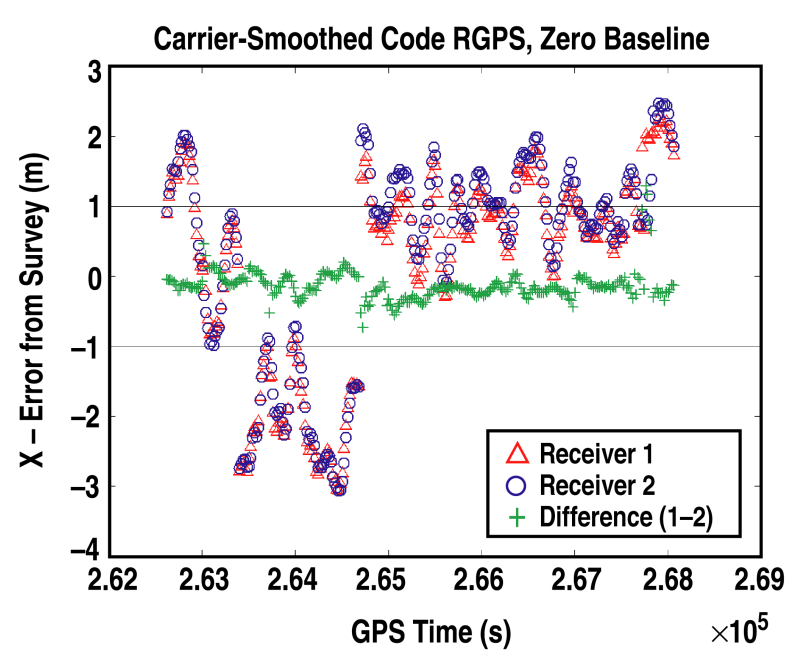

Fig. 3. RGPS with no decorrelation.

fluctuations are highly correlated, and that the error difference between receivers is well below $1 \mathrm{~m}(16 \mathrm{~cm}$ CEP).

Figure 3 shows an idealized case, with both receivers using the same antenna, which is not representative of precision weapon guidance. Physical separation between the sensor and munition causes spatial decorrelation that decreases the commonality of the bias-like errors between their respective GPS solutions. A similar time decorrelation applies to GPS data separated in time. Estimates for both temporal and spatial decorrelation have been proposed in the literature. ${ }^{7,8}$ The spatial predictions show errors below a decimeter for typical sensor-to-target ranges $(10-30 \mathrm{~km})$. The temporal decorrelation is estimated at 30 centimeters for a 20 minute lag. These results imply that temporal vice spatial decorrelation is a more significant concern for this targeting concept. However, these estimates seem to be made from numerical simulations and may not properly account for the decorrelation of bias-like effects of Ionospheric and Tropospheric delays. Experience with carrier phase DGPS has shown increased difficulty converging to solutions beyond 10-20 km. Explanations for this difficulty typically blame the Ionosphere.

RGPS is only a part of the relative guidance problem. RGPS does compensate for the position vector between the GPS antenna on the UAV and the GPS antenna on the munition, but the objective of relative guidance is to direct the weapon onto the target. Therefore it is the munition to target vector that is important. Once the vector from the GPS antenna of the UAV to the target is determined simple vector arithmetic will generate the desired munition to target vector. Four distinct steps are involved in the process to determine the effective munition to target vector:

(1) The only means to determine the target coordinates is the high-performance SAR. If the SAR could measure a precise target location with respect to an instantaneous SAR antenna location, it would provide a SAR antenna to target vector. A stereo SAR algorithm, developed by Sandia National Laboratory, is used to determine target coordinates from a pair of SAR images. Unfortunately, these images cover significant spatial and temporal space, which makes determining a point for the antenna end of the vector problematic. However, the stereo SAR process may be modeled as an integration of the entire set of SAR antenna to target pulses. The sensor already solves for the position of its antenna in an inertial reference frame in order to process SAR imagery. The average of these positions over both apertures will give an average coordinate for the Lynx antenna that can be coupled to the target to form a vector between the sensor and target. SAR measurements are taken with respect to the SAR antenna, instead of the GPS antenna. It is a simple procedure to measure the lever arm between antennas and use that to translate the coordinates, provided that UAV attitude information is available.

(2) A set of correction vectors can then be determined by comparing the values from the SAR navigation solution with those from the RGPS algorithm for the same location. A representative correction vector $(\Delta)$, such as the average of the set, can then be applied to modify the target coordinates and shift them into the RGPS solution space. Figure 4 depicts just such a transformation of solution space applicable to targeting. Note that a solution space is not determined by the coordinate frame, but by the data and algorithm used to formulate the navigation solution. For example, the SAR may use a navigation solution based upon 8 of 10 visible SVs integrated with INS data in a Kalman filter. A separate GPS-only solution that uses all $10 \mathrm{SVs}$ could be defined in the same coordinate frame, but have a significantly different solution for the same location. 


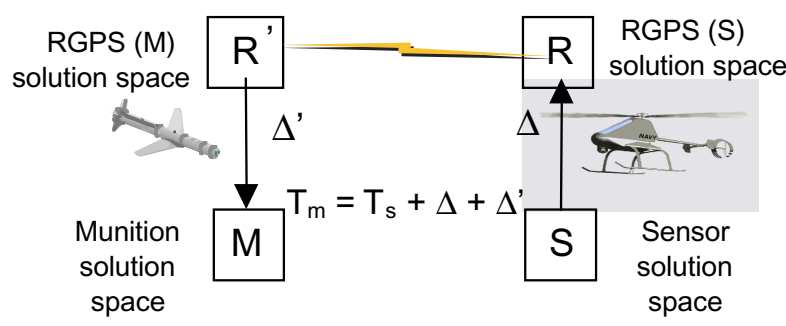

Fig. 4. Solution space transform diagram.

(3) There is no way to directly compare any navigation solution from the munition with that of the sensor at the same location. Therefore both RGPS solution spaces (for the munition and sensor) are designed to be as common as possible. This is accomplished by forcing the solutions to the same algorithm with the same SVs. A datalink between the two platforms is used to determine which SVs are available to both receivers and to pass the modified target coordinates $\left(\mathrm{T}_{\mathrm{r}}-\right.$ those within the RGPS solution space) to the munition.

(4) The final step in determining the munition to target vector is to transform the target coordinates into the munition solution space, $T_{\mathrm{r}}$ to $\mathrm{T}_{\mathrm{m}}$. This step is similar to the second step except that the correction $\left(\Delta^{\prime}\right)$ is updated epoch to epoch. $\Delta^{\prime}$ is computed as the most recent difference between the RGPS solution space (at the munition) and the munition solution space. The equation in Figure 4 shows the complete transformation from the solution space of the sensor to that of the munition.

A major component of error inherent in this relative guidance scheme is the temporal and spatial decorrelation of the GPS solutions. The INS is likely to be very accurate over the 10-20 seconds of low acceleration flight typical of representative SAR apertures. Figure 5 supports this hypothesis. It is a comparison of the sensor height solution with that of an all-in-view GPS receiver height, outputting carrier-smoothed code solutions. Note that $\Delta$ changes very little over the length of the aperture.

\section{FLIGHT DEMONSTRATIONS}

A set of flight experiments were conducted with the goal of establishing a baseline capability for stereo SAR targeting accuracy with relative guidance. The experiments were also designed to help establish the sensitivity of system performance (accuracy) to parameters considered likely to have an impact on performance. The philosophy has been to start with a simple, inexpensive approach and build the lessons learned from early experiments into a prototype system in the future. UAV platforms are typically in high demand. This scarcity and the flexibility of manned commercial aircraft, like the Beech King Air, make their substitution for UAV platforms a sensible decision in the early developmental stages of a program. Examples of this flexibility include the ease with which data can be logged, equipment can be modified, or flights rescheduled while using the commercial airplane.

Two data collection efforts have been conducted at a total of 5 different sites. The first effort occurred during two weeks of July 2001, and the second was two weeks during November 2001.

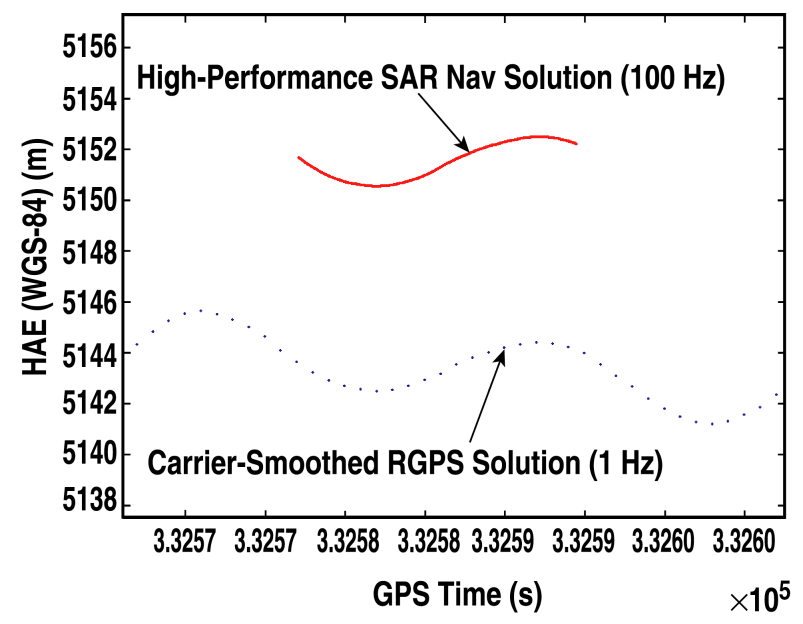

Fig. 5. $\Delta$ is relatively constant over aperture.

\section{Experimental Setup}

The SAR was mounted on a Beech King Air used to simulate the flying conditions of a UAV. In general the primary difference is speed. Apertures were typically collected with groundspeeds on the order of 80-100 m per second (160-190 knots). Many UAV candidates for tactical operation are likely to fly at roughly half that speed. Although some of the newer UAVs can fly faster. No effort has been made to adjust for this effect which is not expected to significantly affect performance. 
Inside the King Air a SAR operator could type in parameters to set up a spotlight aperture on a LCD screen that helped the pilots to navigate to it. The SAR automatically started an aperture when the plane was past the planned start point, and it stored the resulting images. A function was added for the second data collection to enhance the navigation data storage. Three GPS systems were spliced to a GPS patch antenna on top of the plane just aft of the cockpit. One was a military receiver used by the SAR for inclusion in a GPS/INS Kalman filter. The other two recorded data for later processing. Of these two one was an identical military receiver and the other was a commercial receiver. During July, the commercial receiver was a Trimble XRS pro used exclusively for determining carrier-phase DGPS position of the aircraft. During November, the receiver was upgraded to a Novatel OEM4, dual frequency receiver. This unit recorded raw data intended to be post-processed both for carrierphase DGPS and RGPS positions.

A field battery "suitcase" was constructed to allow GPS data collection at remote sites. In July this unit powered another identical military receiver with its antenna located at the "target". An identical Trimble XRS pro was split to the same antenna, although it used its own battery source. In November, a Novatel OEM4 substituted for the Trimble, and was attached to the suitcase battery. Both in the air and on the ground, the military and Novatel receivers recorded their data over serial cables to binary files on laptop PCs. The Trimble units recorded data to a handheld recorder. For both collections, the ground data was recorded with the assumption that it would be similar to the signals received by a munition immediately prior to impact at the target.

\section{Test Flights}

Approximately 30 flight hours were flown in support of Stereo SAR targeting during July. All of this time was spent at a single site with three separate target scenes. The data collection was planned with the intent to perform analysis of variance (ANOVA) upon the following parameters: slant range, $\psi$, and resolution. Two values for each parameter were used for a total of eight " $\mathrm{X}$ " configurations at each scene during the first week.
All ground track separations were set at $20 \mathrm{deg}$. Collection for the second week was identical to the first except for the schedule, which was randomized for both cases.

The November collection was completed with approximately 20 flight hours. For this collection, the ground track separation was varied, with the other parameters constant. Three X configurations were completed for each boresight angle with four primary scenes each at a separate site. Two of the sites used two different boresights. Figure 6 shows an overhead view of a complete collection at one of these sites. The displayed navigation data is that of the sensor solution space, which was collected only during aperture formation.

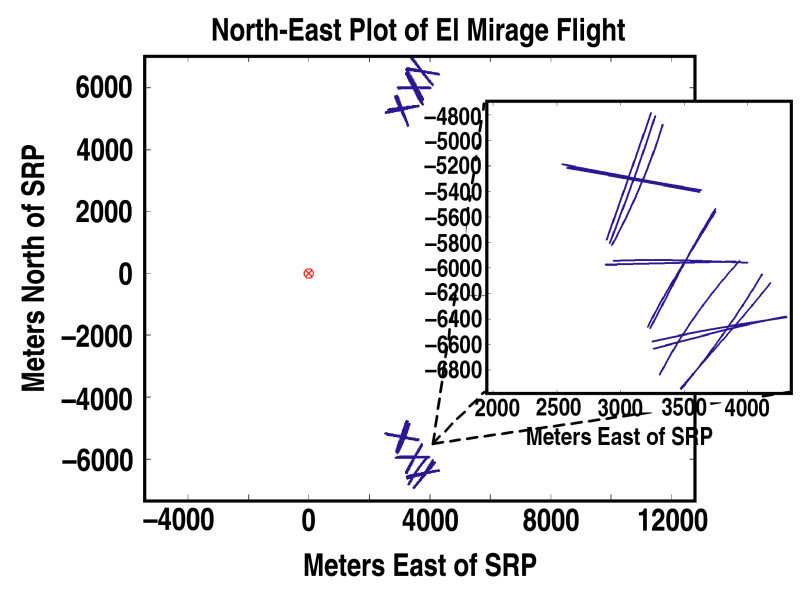

Fig. 6. Typical collection geometry.

\section{Preliminary Results}

A complete and thorough analysis has yet to be completed on either data set. However, several interesting trends have been identified that are worthy of note. The results from a typical stereo pair are also included.

So far the results have been encouraging. Absolute accuracy errors have ranged from single digits to a few tens of meters. The most sensitive parameters seem to be range, resolution, aperture alignment, and the vertical displacement of the focus plane. Crossrange layover is dependent upon the object height relative to the focus plane. As the vertical displacement between the focus plane and the target increases the gross separation of 
crossrange layover also increases. This may lead to a decrease in the stereo correlation, and eventually cause problems due to depth of focus limitations.

Early results with sub-optimal RGPS show some accuracy improvement over straight absolute coordinates in most cases. The ground receiver data solved with the RGPS algorithm and a common set of SVs gives the correct target coordinates in the RGPS solution space $\left(\mathrm{T}_{\mathrm{r} 0}\right)$. Accuracy results were obtained by adding the $\Delta$ correction to the computed stereo SAR coordinates $\left(\mathrm{T}_{\mathrm{S}}\right)$ and then comparing the resulting coordinates $\left(\mathrm{T}_{\mathrm{r}}\right)$ to coordinates from a pre-selected ground epoch $\left(\mathrm{T}_{\mathrm{r} 0}\right)$. The $\mathrm{T}_{\mathrm{r} 0}$ epoch was selected as a reasonable time to expect for munition impact. Horizontal accuracy improvement of the relative error versus absolute error is near universal, but the relative vertical error often increased. This may be due to an undetermined systematic error.

Figures 7 and 8 form the 4 -in. resolution stereo SAR image pair used to create Figs. 9 and 10, an orthorectified image and DEM respectively. The stereo pair was imaged at a slant range of $9 \mathrm{~km}$, with $\psi$ of $30 \mathrm{deg}$, and a ground track separation of $60 \mathrm{deg}$. A small circle in each image indicates the location of the GPS antenna.

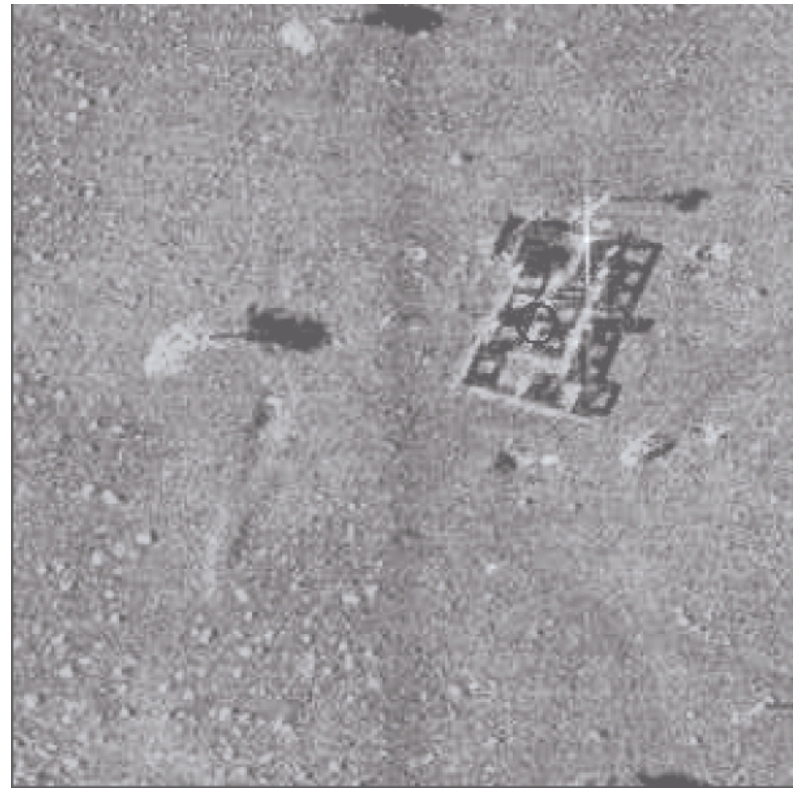

Fig. 7. First image of stereo pair.
The agreement between the horizontal target coordinates as transformed into RGPS solution space with the ground GPS coordinates was excellent. A significant difference in the vertical coordinates may be partially due the poor performance of the algorithm in capturing the vertical walls of the abandoned building. A reasonable delay was used in choosing the ground coordinates for comparison.

\section{SUMMARY}

An innovative concept to generate accurate and timely target coordinates was introduced. The technique can be used in poor weather conditions that preclude targeting by traditional means. A technique for performing spotlight Stereo SAR coordinate extraction was described. Relative guidance based upon RGPS and the transformation between navigational solution spaces was also presented. Flight testing of the concept in semi-dynamic conditions (using a static mock weapon) produced encouraging results that have not been fully explored.

\section{ACKNOWLEDGEMENTS}

The authors would like to give thanks to Don Small, Mark Dowdican, and Ted Kim of Sandia National Laboratories for their contributions relating to use of

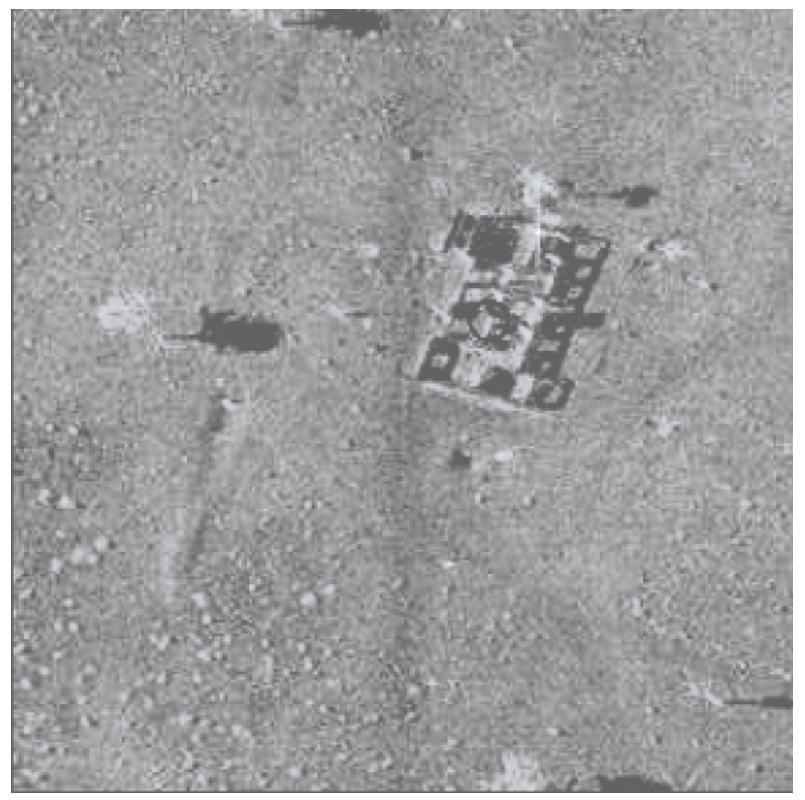

Fig. 8. Second image of stereo pair. 


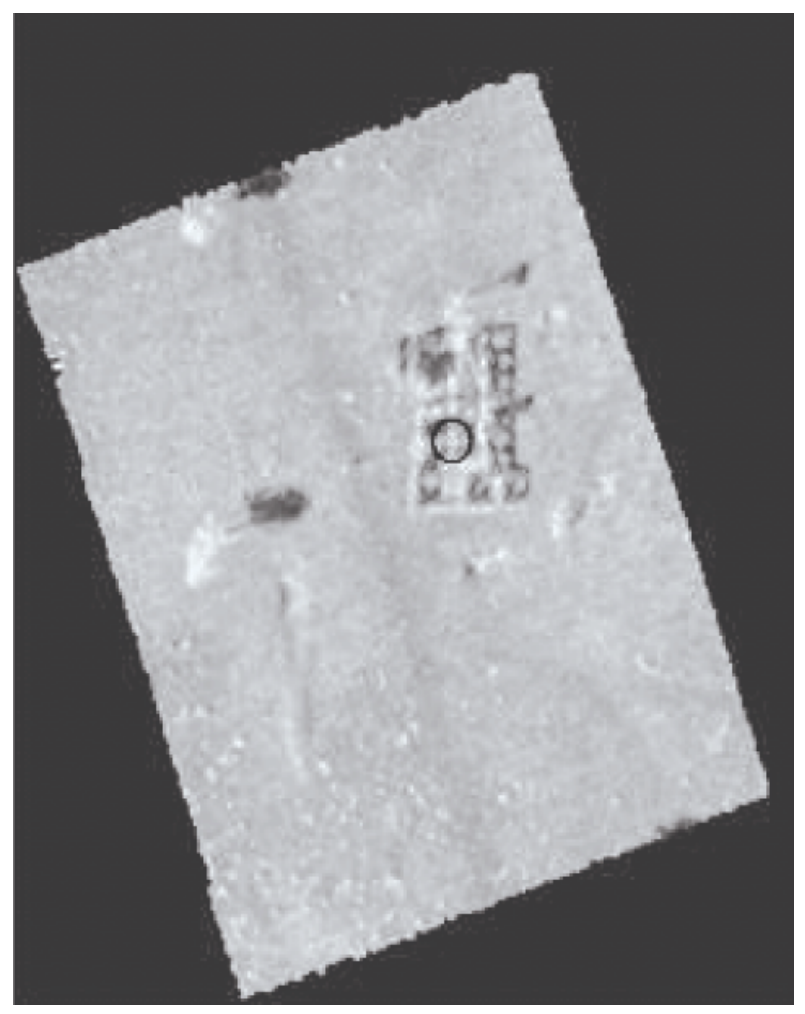

Fig. 9. Orthorectified product.

the stereo SAR algorithm and the navigational solution of the sensor, and also to Ed Weston of Naval Air Warfare Center, China Lake for help troubleshooting the RGPS algorithm. The authors are grateful to James Chew and Gil Graff of the Office of Naval Research, and John Heidt and Bob Sutton of Naval Air Warfare Center, China Lake who have served as program managers and have supported this work.

This is a report of work supported by the U.S. Naval Air Warfare Center under Contract No. N68936-01-C0028 .

\section{REFERENCES}

${ }^{1}$ Schmidt, G., "GPS/INS Trends for Military Systems," 97-3826 AIAA Guidance, Navigation, and Control Conference, New Orleans, LA, August 11-13, 1997.

${ }^{3}$ Koopmans, B., "Should Stereo SLAR Imagery be Preferred to Single Strip Imagery for Thematic Mapping?," ITC-Journal 1974-3, Enschede, The Netherlands.

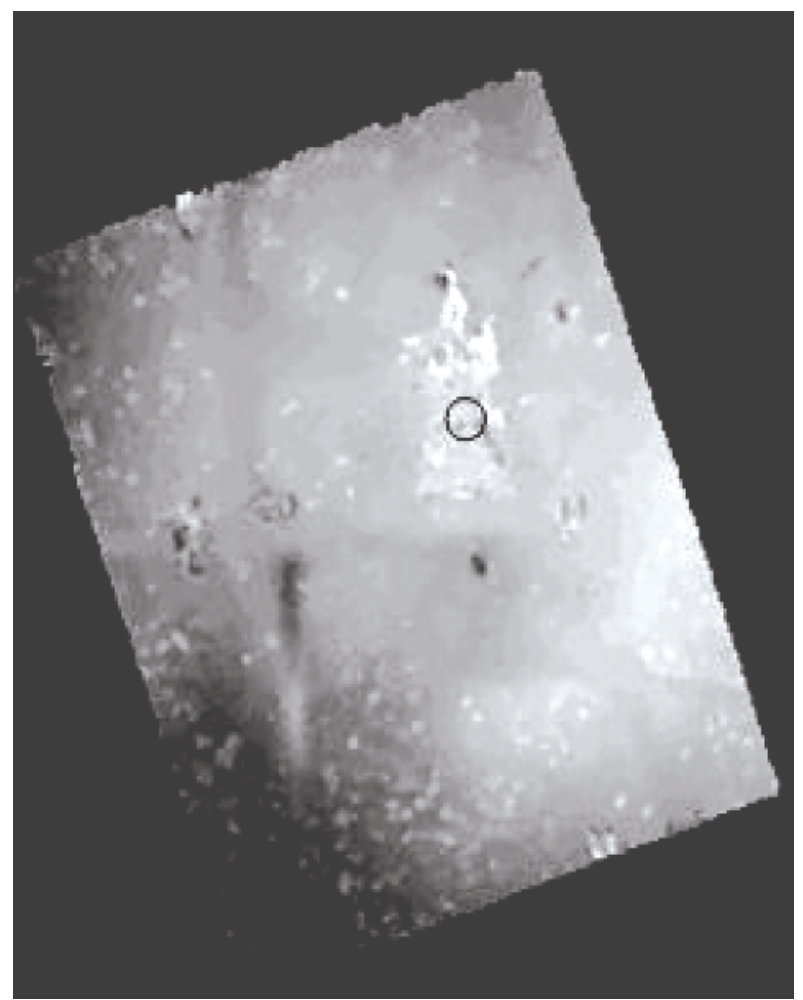

Fig. 10. DEM product of target.

${ }^{2}$ Schmidt, G., Setterlund, R., "Precision Strike Concepts Exploiting Relative GPS Techniques," Navigation, Journal of the Institute of Navigation, Vol. 41, No. 1 (1994).

${ }^{4}$ Jakowatz, C., et al., Spotlight Mode Synthetic Aperture Radar: a Signal Processing Approach (Kluwer Academic Publishers, Norwell, 1996).

5Leberl, F.W., Radargrammetric Image Processing (Artech House, Norwood, 1990).

${ }^{6}$ Misra, P., Burke, B., and Pratt, M., "GPS Performance in Navigation," Proceedings of the IEEE, Vol. 87, No. 1 (1999).

${ }^{7}$ Youhanaie, M., Hartman, D., Ziolkowski, F., Pujara, N., "Robust Implementation of Relative GPS Guidance," Institute of Navigation, Proceedings of the National Technical Meeting, 1995.

${ }^{8}$ Phillips, R., "Relative and Differential GPS Data Transfer and Error Analysis," Proceedings of the 53rd Annual Meeting, Institute of Navigation, Alexandria, VA, pp. 735-741 (1997). 